\title{
HOMOLOGY GROUP ON MANIFOLDS AND THEIR FOLDINGS
}

\author{
M. ABU-SALEEM
}

\begin{abstract}
In this paper, we introduce the definition of the induced unfolding on the homology group. Some types of conditional foldings restricted on the elements of the homology groups are deduced. The effect of retraction on the homology group of a manifold is dicussed. The unfolding of variation curvature of manifolds on their homology group are represented. The relations between homology group of the manifold and its folding are deduced.
\end{abstract}

\section{Introduction}

The folding of a manifold introduced by S. A. Robertson 1977 [13]. More studies of the folding of manifolds are studied in $[4,5,7,11,14,15]$. Various folding problems arising in the physics of membrane and polymers reviewed by Francesco [2]. The unfolding of a manifold introduced in [3]. The retraction of a manifold defined and discussed in [6, 12]. The homology groups of some types of a manifold are discussed in $[1,8,9,10,16]$.

\section{Definitions and background}

In this section, we give the definitions which are needed especially in this paper.

Definition 1. Consider the sequence $C_{p+1}(k) \stackrel{\partial_{p+1}}{\longrightarrow} C_{p}(k) \stackrel{\partial_{p}}{\longrightarrow} C_{p-1}(k)$, ker $\partial_{p}$ is denoted by $Z_{p}(K)$, and the elements of $Z_{p}(K)$ are called $p$-cycles, $I m \partial_{p+1}$ is denoted by $B_{p}(K)$, and the elements of $B_{p}(K)$ are called $p$-boundaries. The quotient group $H_{p}=Z_{p}(K) / B_{p}(K)$ is called the $P^{t h}$ homology group of $K$ [8].

Definition 2. Let $M$ and $N$ be two manifolds of dimensions $m$ and $n$ respectively. A map $f: M \rightarrow N$ is said to be an isometric folding of $M$ into $N$ if for every piecewise geodesic path $\gamma: I \rightarrow M$, the induced path $f \circ \gamma: I \rightarrow N$ is piecewise geodesic and of the same length as $\gamma$ [13]. If $f$ does not preserve length, it is called a topological folding [11].

Received February 18, 2009.

2000 Mathematics Subject Classification. 51H20, 55N35, 14F35.

Key words and phrases. Manifolds, folding, homology group. 
Definition 3. Let $M$ and $N$ be two manifolds of the same dimension. A map $g: M \rightarrow N$ is said to be unfolding of $M$ into $N$ if, for every piecewise geodesic path $\gamma: I \rightarrow M$, the induced path $g \circ \gamma: I \rightarrow N$ is piecewise geodesic with length greater than $\gamma[3]$.

Definition 4. A subset $A$ of a topological space $X$ is called a retract of $X$ if there exists a continuous map $r: X \rightarrow A$ (called a retraction) such that $r(a)=a \forall a \in A$ [12].

Definition 5. Given spaces $X$ and $Y$ with chosen points $x_{0} \in X$ and $y_{0} \in Y$, then the wedge sum $X \vee Y$ is the quotient of the disjoint union $X \cup Y$ obtained by identifying $x_{0}$ and $y_{0}$ to a single point [9].

\section{The main results}

Aiming to our study, we will introduce the following:

Definition 6. Let $M$ and $M$ be two manifolds of the same dimensions and unf:M $\rightarrow$ $\dot{M}$ be any unfoding of $M$ into $M$. Then, a map $\overline{u n f}: H_{n}(M) \rightarrow H_{n}(\dot{M})$ is said to be an induced unfolding of $H_{n}(M)$ into $H_{n}\left(M^{\prime}\right)$ if $\overline{u n f}\left(H_{n}(M)\right)=H_{n}(u n f(M))$.

Lemma 1. Let $C_{1}, C_{2}$ be two disjoint circles in $R^{2}$. Then there is unfolding unf: $C_{1} \cup C_{2} \rightarrow \dot{C}_{1} \cup \dot{C}_{2}$ which induces unfolding

$\overline{u n f}: H_{n}\left(C_{1} \cup C_{2}\right) \rightarrow H_{n}\left(\dot{C}_{1} \cup \dot{C}_{2}\right)$ such that

(1) $\overline{u n f}\left(H_{0}\left(C_{1} \cup C_{2}\right)\right) \approx Z$

(2) $\overline{u n f}\left(H_{1}\left(C_{1} \cup C_{2}\right)\right) \approx Z \oplus Z$

(3) $\left.\overline{u n f}\left(H_{n} C_{1} \cup C_{2}\right)\right) \approx 0$, for $n \geq 2$.

Proof. Let unf: $C_{1} \cup C_{2} \rightarrow \dot{C}_{1} \cup \dot{C}_{2}$ be unfolding such that

unf $\left(C_{1} \cup C_{2}\right)=\operatorname{unf}\left(C_{1}\right) \vee \operatorname{unf}\left(C_{2}\right)$ as in Figure 1, thus we get the induced unfolding $\overline{u n f}: H_{n}\left(C_{1} \cup C_{2}\right) \rightarrow H_{n}\left(\dot{C}_{1} \cup \dot{C}_{2}\right)$ such that $H_{n}\left(\right.$ unf $\left.\left(C_{1} \cup C_{2}\right)\right)=H_{n}\left(\right.$ unf $\left(C_{1}\right) \vee$ unf $\left.\left(C_{2}\right)\right)$.

Now, for $n=0, \overline{u n f}\left(H_{0}\left(C_{1} \cup C_{2}\right)\right)=H_{0}\left(u n f\left(C_{1} \cup C_{2}\right)\right) \approx Z$. Also, if $n=$ $1, \overline{u n f}\left(H_{1}\left(C_{1} \cup C_{2}\right)\right)=H_{1}\left(\right.$ unf $\left.\left(C_{1} \cup C_{2}\right)\right) \approx H_{1}\left(\right.$ unf $\left.\left(C_{1}\right)\right) \oplus H_{1}\left(\right.$ unf $\left.\left(C_{2}\right)\right)$. Since $H_{1}\left(\operatorname{unf}\left(C_{i}\right)\right) \approx Z, i=0,1$ it follows that $\overline{u n f}\left(H_{1}\left(C_{1} \cup C_{2}\right)\right) \approx Z \oplus Z$.
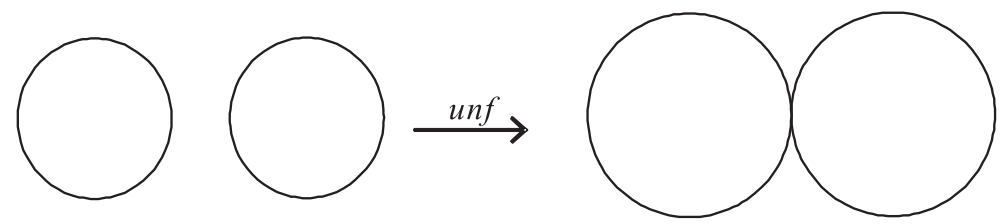

Figure 1: 
Moreover, if $n \geq 2$, it follows from $H_{n}\left(\right.$ unf $\left.f\left(C_{i}\right)\right) \approx 0, i=0,1$ and from $H_{n}\left(\right.$ unf $\left(C_{1} \cup\right.$ $\left.\left.C_{2}\right)\right) \approx H_{n}\left(u n f\left(C_{1}\right)\right) \oplus H_{n}\left(\right.$ unf $\left.\left(C_{2}\right)\right)$ that $\overline{u n f}\left(H_{n}\left(C_{1} \cup C_{2}\right)\right)=H_{n}\left(u n f\left(C_{1} \cup C_{2}\right)\right) \approx 0$, for $n \geq 2$.

Lemma 2. Let I be the closed interval $[0,1]$ and let $\bar{I}$ be the closed interval $[-1,0]$. Then there are unfoldings unf $f_{m}: I \cup \bar{I} \rightarrow J \cup \bar{J} m=1,2, \ldots$ with variation curvature which induces unfoldings $\overline{u n f_{m}}: H_{n}(I \cup \bar{I}) \rightarrow H_{n}(J \cup \bar{J}), m=1,2, \ldots$ such that

(1) $\lim _{m \rightarrow \infty}\left(\overline{u n f_{m}}\left(H_{0}(I \cup \bar{I})\right)\right) \approx Z$

(2) $\lim _{m \rightarrow \infty}\left(\overline{u n f_{m}}\left(H_{1}(I \cup \bar{I})\right)\right) \approx Z \oplus Z$

(3) $\lim _{m \rightarrow \infty}\left(\overline{u n f_{m}}\left(H_{n}(I \cup \bar{I})\right)\right) \approx 0$, for $n \geq 2$.

Proof. Consider the sequence of unfoldings with variation curvature such that $u f_{1}$ : $I \cup \bar{I} \rightarrow X_{1}$, unf $_{2}: X_{1} \rightarrow X_{2}, \ldots$, unf $_{m}: X_{m-1} \rightarrow X_{m}$ such that $\lim _{m \rightarrow \infty}\left(u n f_{m}(I \cup \bar{I})\right)=S_{1}^{1} \vee S_{2}^{1}$ as in Figure 2, thus we get the induced unfoldings $\frac{m \rightarrow \infty}{u n f_{m}}: H_{n}(I \cup \bar{I}) \rightarrow H_{n}(J \cup \bar{J}), m=1,2, \ldots$ such that $\lim _{m \rightarrow \infty}\left(\overline{u n f_{m}}\left(H_{n}(I \cup \bar{I})\right)\right)=$ $H_{n}\left(\lim _{m \rightarrow \infty}\left(u n f_{m}(I \cup \bar{I})\right)\right)$. Now, for $n=0, \lim _{m \rightarrow \infty}\left(\overline{u n f_{m}}\left(H_{0}(I \cup \bar{I})\right)\right)=H_{0}\left(\lim _{m \rightarrow \infty}\left(u n f_{m}(I \cup\right.\right.$ $\bar{I})))=H_{0}\left(S_{1}^{1} \vee S_{2}^{1}\right) \approx Z$. Also, if $n=1$, it follows from $H_{1}\left(S_{1}^{1} \vee S_{2}^{1}\right) \approx H_{1}\left(S_{1}^{1}\right) \oplus$ $H_{1}\left(S_{2}^{1}\right)$, and from $H_{1}\left(S_{i}\right) \approx Z, i=1,2$ that

$\lim _{m \rightarrow \infty}\left(\overline{u n f_{m}}\left(H_{1}(I \cup \bar{I})\right)\right)=H_{1}\left(\lim _{m \rightarrow \infty}\left(u n f_{m}(I \cup \bar{I})\right)\right) \approx Z \oplus Z$. Moreover, if $n \geq 2$, it follows from $H_{n}\left(S_{1}^{1} \vee S_{2}^{1}\right) \approx H_{n}\left(S_{1}^{1}\right) \oplus H_{n}\left(S_{2}^{1}\right)$, and from $H_{n}\left(S_{i}\right) \approx 0, i=1$, 2 , that $\lim _{m \rightarrow \infty}\left(\overline{u n f_{m}}\left(H_{n}(I \cup \bar{I})\right)\right)=H_{n}\left(\lim _{m \rightarrow \infty}\left(u n f_{m}(I \cup \bar{I})\right)\right) \approx 0$. Therefore we get the induced unfoldings $\overline{u n f_{m}}: H_{n}(I \cup \bar{I}) \rightarrow H_{n}(J \cup \bar{J}), m=1,2, \ldots$ such that

(1) $\lim _{n \rightarrow \infty}\left(\overline{u n f_{m}}\left(H_{0}(I \cup \bar{I})\right)\right) \approx Z$
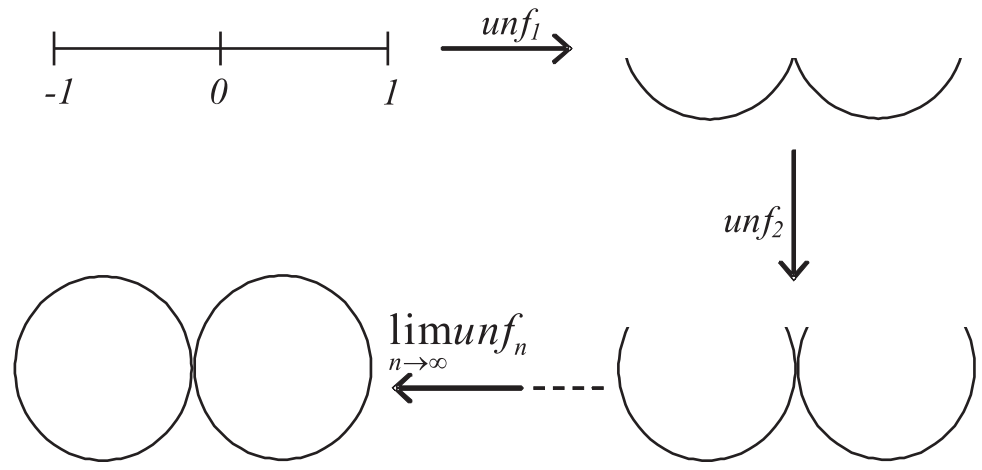

Figure 2: 
(2) $\lim _{n \rightarrow \infty}\left(\overline{u n f_{m}}\left(H_{1}(I \cup \bar{I})\right)\right) \approx Z \oplus Z$

(3) $\lim _{n \rightarrow \infty}\left(\overline{u n f_{m}}\left(H_{n}(I \cup \bar{I})\right)\right) \approx 0$, for $n \geq 2$.

Theorem 1. Let $C$ be the circle of radius 1 and centre $(-1,0)$. Then there are infinite number of unfoldings unf $f_{m}: C \vee \bar{C} \rightarrow C_{1} \vee \bar{C}_{1}, m=1,2, \ldots$ which induces unfoldings $\overline{u n f}_{m}: H_{n}(C) \oplus H_{n}(\bar{C}) \rightarrow H_{n}\left(C_{1}\right) \oplus H_{n}\left(\bar{C}_{1}\right), n \geq 1$ such that

$\overline{u n f}_{m}\left(H_{n}(C) \oplus H_{n}(\bar{C})\right)=H_{n}\left(u n f_{m}(C)\right) \oplus H_{n}\left(u n f_{m}(\bar{C})\right)$ or

$\overline{u n f}_{m}\left(H_{n}(C) \oplus H_{n}(\bar{C})\right)=H_{n}\left(u n f_{m}(C)\right) \oplus H_{n}(\bar{C})$ or

$\overline{u n f}_{m}\left(H_{n}(C) \oplus H_{n}(\bar{C})\right)=H_{n}(C) \oplus H_{n}\left(u n f_{m}(\bar{C})\right)$.

Proof. Let $u n f_{m}: C \vee \bar{C} \rightarrow C_{1} \vee \bar{C}_{1}, m=1,2, \ldots$ are unfoldings which are preserving curvature or not preserving curvature such that un $f_{m}(C \vee \bar{C})=u n f_{m}(C) \vee$ un $f_{m}(\bar{C})$ where un $f_{m}(C)$ is the circle of radius $m$ and center $(m, 0)$ and $u n f_{m}(\bar{C})$ is the circle of radius $m$ and center $(-m, 0)$ as in Figure 3 . So we have an induced unfolding $\overline{u n f}_{m}: H_{n}(C) \oplus$ $H_{n}(\bar{C}) \rightarrow H_{n}\left(C_{1}\right) \oplus H_{n}\left(\bar{C}_{1}\right), n \geq 1$ such that $\overline{u n f}_{m}\left(H_{n}(C) \oplus H_{n}(\bar{C})\right)=\overline{u n f}_{m}\left(H_{n}(C)\right) \oplus$ $\left.\overline{u n f}_{m} H_{n}(\bar{C})\right)$, thus $\overline{u n f}_{m}\left(H_{n}(C) \oplus H_{n}(\bar{C})\right)=H_{n}\left(u n f_{m}(C)\right) \oplus H_{n}\left(\right.$ unf $\left._{m}(\bar{C})\right)$. Similarly, we can get the induced unfolding $\overline{u n f}_{m}: H_{n}(C) \oplus H_{n}(\bar{C}) \rightarrow H_{n}\left(C_{1}\right) \oplus H_{n}\left(\bar{C}_{1}\right)$ such that $\overline{u n f}_{m}\left(H_{n}(C) \oplus H_{n}(\bar{C})\right)=H_{n}\left(u n f_{m}(C)\right) \oplus H_{n}(\bar{C})$ or $\overline{u n f}_{m}\left(H_{n}(C) \oplus H_{n}(\bar{C})\right)=H_{n}(C) \oplus H_{n}\left(u n f_{m}(\bar{C})\right)$.

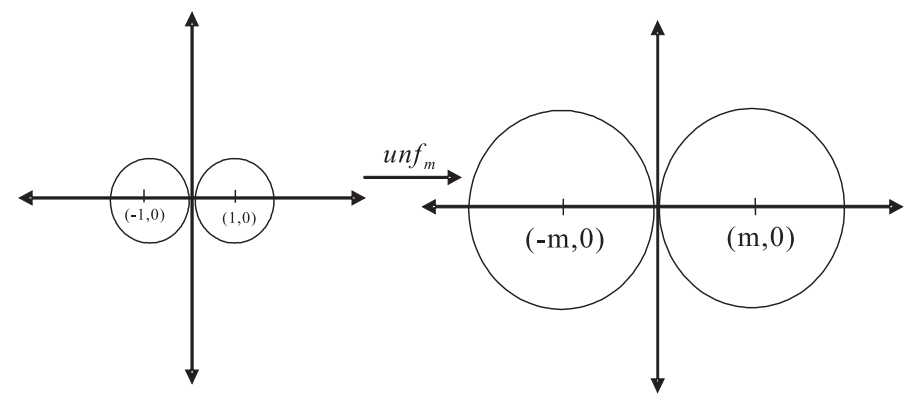

Figure 3:

Theorem 2. Let $X$ be the subspace of $R^{2}$ that is the union of the circles $C_{m}$ of radii $\frac{1}{m}$ and centres $\left(\frac{1}{m}, 0\right)$ for $m=1,2, \ldots$, then there is unfolding unf $: X \rightarrow \bar{X}$ which induced

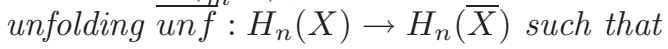

(1) $\overline{u n f}\left(H_{0}(X)\right) \approx Z$

(2) $\overline{u n f}\left(H_{1}(X)\right)$ is a free abelian group on a countable set of generators.

(3) $\overline{u n f}\left(H_{n}(X)=0\right.$, for $n \geq 2$.

Proof. Let unf $X \rightarrow \bar{X}$ be unfolding such that $\operatorname{unf}(X)=\operatorname{unf}\left(\bigcup_{m=1}^{\infty} C_{m}\right)=\bigcup_{m=1}^{\infty}$ unf $\left(C_{m}\right)$ and $\operatorname{unf}\left(C_{m}\right)$ is the circles of radius $m$ and centre $(m, 0)$ as in Figure ??. Then we get the induced unfolding $\overline{u n f}: H_{n}(X) \rightarrow H_{n}(\bar{X})$ such that $\overline{u n f}\left(H_{n}(X)\right)=H_{n}(u n f(X))$. 
Thus for $n=0, \overline{u n f}\left(H_{0}(X)\right)=H_{0}(u n f(X)) \approx Z$.

Now, for $n=1$ we want to show that, $H_{1}(u n f(X))$ is a free group on a countable set of generators. It follows from $\operatorname{unf}(X)=\bigcup_{m=1}^{\infty} \operatorname{unf}\left(C_{m}\right)$ that $\operatorname{unf}(X)$ is the union of the circles of radius $m$ and center $(m, 0)$, since $u n f(X)$ and $\vee S^{1}$ are homotopy equivalent, we have $H_{1}($ unf $f(X)) \approx H_{1}\left(\bigvee_{\infty}^{\vee} S^{1}\right)$ and so $H_{1}($ unf $\left.f(X)) \approx H_{1}(Z) \oplus H_{1}(Z) \oplus \cdots \infty\right)$. Therefore, $\overline{u n f}_{m}\left(H_{1}(X)\right)$ is a free abelian group on a countable set of generators. Moreover, for $n \geq 2$. Clearly we can get $\overline{u n f}\left(H_{n}(X)=0\right.$.

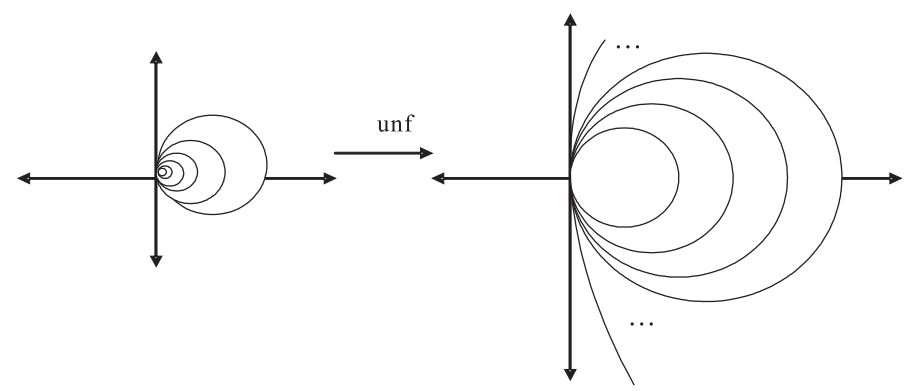

Figure 4:

Theorem 3. Let $X$ be the subspace of $R^{2}$ that is the union of the circles $C_{m}$ of radius $m$ and center $(m, 0)$ for $m=1,2, \ldots$. Then there is a folding $F: X \rightarrow \bar{X}$ without singularity, which induces a folding $\bar{F}: H_{n}(X) \rightarrow H_{n}(\bar{X})$ such that

(1) $\bar{F}\left(H_{0}(X)\right) \approx Z$.

(2) $\bar{F}\left(H_{1}(X)\right)$ is uncountable.

(3) $\bar{F}\left(H_{n}(X)\right)=0$, for $n \geq 2$.

Proof. Let $F: X \rightarrow \bar{X}$ be a folding without singularity such that $F(X)=F\left(\bigcup_{m=1}^{\infty} C_{m}\right)=$ $\bigcup_{m=1}^{\infty} F\left(C_{m}\right)$ and $F\left(C_{m}\right)$ is the circle of radius $\frac{1}{m}$ and centre $\left(\frac{1}{m}, 0\right)$ as in Figure 5.

Then the induced folding $\bar{F}: H_{n}(X) \rightarrow H_{n}(\bar{X})$ satisfies $\bar{F}\left(H_{n}(X)\right)=H_{n}(F(\bar{X}))$. Thus for $n=0, \bar{F}\left(H_{0}(X)\right)=H_{0}(F(\bar{X})) \approx Z$. Now, for $n=1$ we want to shows that $H_{1}(F(X))$ is uncountable. Consider the retraction $r_{m}: F(X) \rightarrow F\left(C_{m}\right)$ which collapsing all $F\left(C_{i}\right)$ except $F\left(C_{m}\right)$ to origin. Each $r_{m}$ induces a surjection. $\bar{r}_{m}: H_{1}(F(X)) \rightarrow$ $H_{1}\left(F\left(C_{m}\right)\right) \approx Z$, where the origin is a base point. Then the product of the $\bar{r}_{m}$ is a homomorphism $r: H_{1}(F(X)) \rightarrow \prod_{\infty} Z$ to the direct product of infinite number of copies of $Z$ and clearly, we can prove that $r$ is onto, it follows from $\prod_{\infty} Z$ uncountable that $H_{1}(F(X))$ is uncountable. Since $\bar{F}\left(H_{1}(X)\right)=H_{1}(F(X))$ it follows that $\bar{F}\left(H_{1}(X)\right)$ is uncountable. Moreover, for $n \geq 2$. Clearly we can get $\bar{F}\left(H_{n}(X)\right)=0$, for $n \geq 2$. 


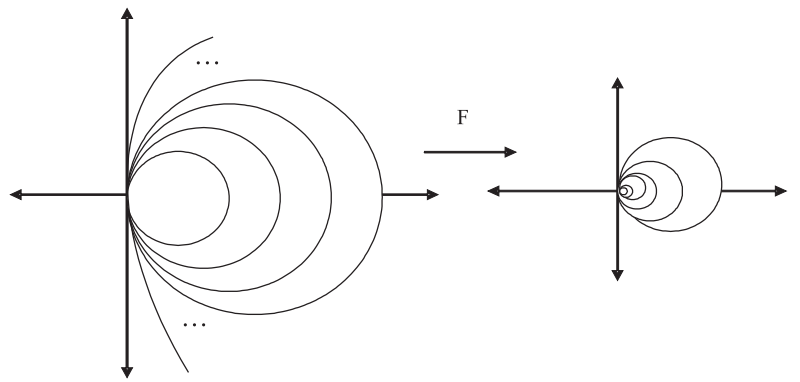

Figure 5:

Theorem 4. Let $X$ be the subspace of $R^{2}$ that is the union of the circles $C_{m}$ of radius $\frac{1}{m}$ and centre $\left(\frac{1}{m}, 0\right)$ for $m=1,2, \ldots$ And $F: X \rightarrow X$ is a folding such that $F\left(C_{m}\right) \neq C_{m}$ $\forall m$. Then there are unfoldings unf $: F(X) \subset X \rightarrow X$ such that $H_{n}\left(\lim _{m \rightarrow \infty}\left(u n f_{m}(F(X))\right)\right.$ is uncountable.

Proof. Let $F: X \rightarrow X$ be a folding such that $F\left(C_{m}\right) \neq C_{m} \forall m$, i.e. folding by cut. Then, we can define a sequence of unfoldings unf $f_{1}: F(X) \rightarrow X_{1}, F(X) \subseteq X_{1} \subseteq$ $X$, unf $_{2}: X_{1} \rightarrow X_{2}, X_{1} \subseteq X_{2} \subseteq X, \ldots$, unf $_{m}: X_{m-1} \rightarrow X_{m}, X_{m-1} \subseteq X_{m} \subseteq X$, and so $\lim _{m \rightarrow \infty}\left(u n f_{m}(F(X))=X\right.$, as in Figure 6, thus $H_{n}\left(\lim _{m \rightarrow \infty}\left(u n f_{m}(F(X))\right)\right.$ is uncountable.

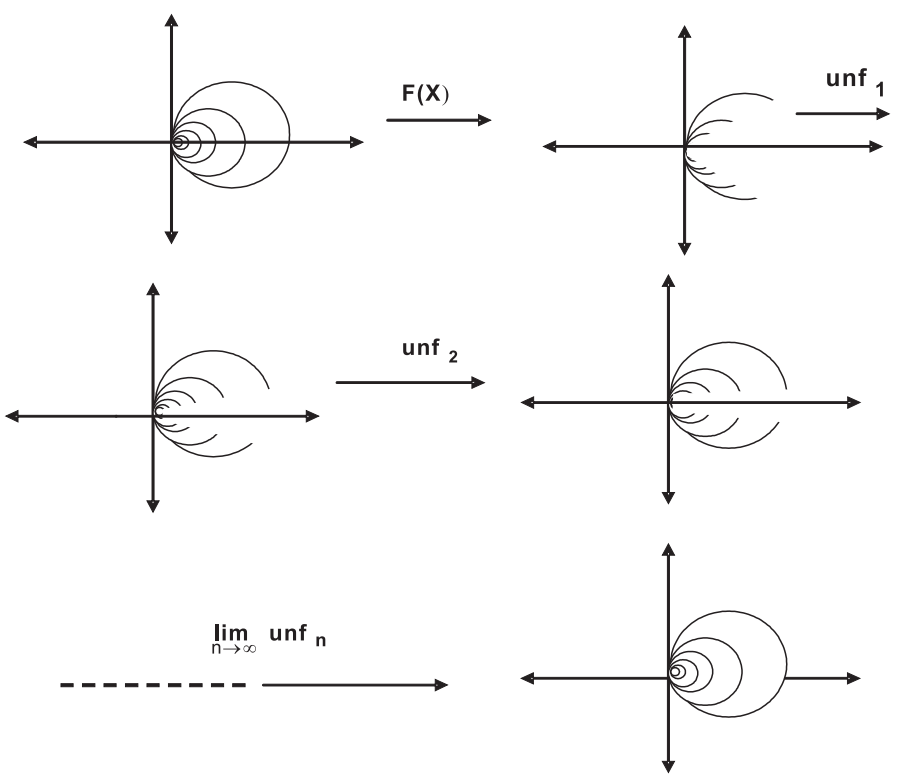

Figure 6: 
Theorem 5. Let $X \subset R^{3}$ be the union of the circles $C_{m}$ of radius $\frac{1}{m}$ and centred at $\left(\frac{1}{m}, 0\right)$ for $m=1,2, \ldots$ Then there are foldings $F_{m}: X \rightarrow X$ and retractions $r_{m}: X \rightarrow$ $C_{m}$ such that $H_{n}\left(F_{m}(X)\right)=H_{n}\left(r_{m}(X)\right)$ and $H_{n}\left(F_{m}(X)\right)$ is either 0 or isomorphic to $Z$.

Proof. Let $F_{m}: X \rightarrow X$ be a folding such that $F_{m}\left(C_{m}\right)=C_{m} \forall m=1,2, \ldots$ then $F_{m}(X)=C_{m}$ as in Figure ?? and so $H_{n}\left(F_{m}(X)\right)=H_{n}\left(C_{m}\right)$. Also, consider the retractions $r_{m}: X \rightarrow C_{m}$, which collapsing all $C_{i}$ except $C_{m}$ to the origin and so $r_{m}(X)=C_{m}$, thus $H_{n}\left(r_{m}(X)\right)=H_{n}\left(C_{m}\right)$. Therefore $H_{n}\left(F_{m}(X)\right)=H_{n}\left(r_{m}(X)\right)$. Now, if $n=0,1$ we have $H_{n}\left(F_{m}(X)\right) \approx Z$.

Also, if $n \geq 2$. we get $H_{n}\left(F_{m}(X)\right) \approx 0$.

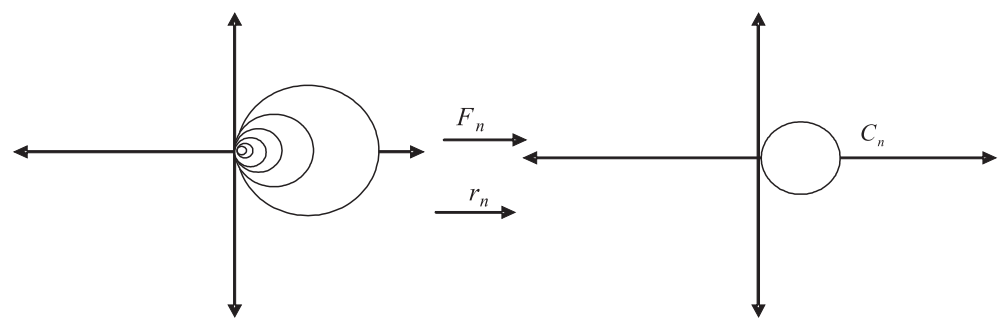

Figure 7:

\section{References}

[1] G. Damiond, S. Peltier and L. Fuchs, Computing homology generators for volumes using minimal generralized maps, In: proceeding of 12 th IWCIA, Volume 4959 of LNCS, USA (2008), 63-74.

[2] P. DI-Francesco, Folding and coloring problem in mathematics and physics, Bulletin of the American Mathematical Society 37 (2000), 251-307.

[3] M. El-Ghoul, Unfolding of Riemannian manifolds, Commun. Fac. Sci. Univ Ankara Series, A 37 (1988), 1-4.

[4] M. El-Ghoul, Fractional folding of a manifold, Chaos, Solitons and Fractals, U.K. 12(2001), 1019-1023.

[5] M. El-Ghoul, A. E. El-Ahmady, H. Rafat and M. Abu-Saleem, The fundamental group of the connected sum of manifolds and their foldings, Chungcheong Mathematical Society 18(2005), 161-172.

[6] M. El-Ghoul, A. E. El-Ahmady, H. Rafat and M. Abu-Saleem, Foldings and retractions of manifolds and their fundamental groups, International Journal of Pure and Applied Mathematics 29(2006), 385-392.

[7] M. El-Ghoul, A. E. El-Ahmady and M. Abu-Saleem, Folding on the Cartesian product of manifolds and their fundamental group, Applied Sciences 9(2007), 86-91.

[8] P. J. Giblin, Graphs surfaces and homology: An introduction to Algebraic topology, Canada, Jon Wiley \& Sons, New York, 1977.

[9] A. Hatcher, Algebraic topology, The web addressis : http//www . math . coronell. edu/hatcher. 
[10] S. T. Hu, Homology theory, Holden-day Inc., San Francisco, 1966.

[11] E. El-Kholy, Isometric and topological folding of manifold, Ph. D. Thesis, University of Southampton, UK., 1981.

[12] W. S. Massey, Algebraic topology: An introduction, Harcourt Brace and world, New York, 1967.

[13] S. A. Robertson, Isometric folding of Riemannian manifolds, Proc. Roy. Soc. Edinburgh 77 (1977), 275-289.

[14] M. Abu-Saleem, Some geometric transformations on manifolds and their algebraic structures, Ph.D. Thesis, University of Tanta, Egypt, 2007.

[15] M. Abu-Saleem, Folding on the chaotic Cartesian product of manifolds and their fundamental group, Tamkang Journal of Mathematics 39 (2008), 353-361.

[16] H. El -Zohny, Homology and chaotic unfolding of chaos manifolds, Indian J. Pure Appl. Math. 35 (2004), 51-55.

Department of Mathematics, Al-Laith college for girls,Umm AL-Qura University, Saudi Arabia.

E-mail: mohammedabusaleem2005@yahoo.com 